\title{
The DESI spectrograph system and production
}

Jerry Edelstein, Patrick Jelinsky, Michael Levi, Gregory Tarle, David Brooks

Jerry Edelstein, Patrick Jelinsky, Michael Levi, Gregory Tarle, David Brooks, "The DESI spectrograph system and production," Proc. SPIE 10702, Ground-based and Airborne Instrumentation for Astronomy VII, 107027G (9 July 2018); doi: 10.1117/12.2311821

SPIE Event: SPIE Astronomical Telescopes + Instrumentation, 2018, Austin, Texas, United States 


\title{
The DESI Spectrograph System and Production
}

\author{
Jerry Edelstein ${ }^{\star \mathrm{a}}$, Patrick Jelinsky ${ }^{\mathrm{a}}$, Michael Levi ${ }^{\mathrm{b}}$, Gregory Tarle ${ }^{\mathrm{c}}$ and David Brooks ${ }^{\mathrm{d}}$, for \\ the DESI Collaboration \\ ${ }^{a}$ Spaces Sciences Laboratory, University of California, 7 Gauss Way, Berkeley, CA, USA \\ 94720; ${ }^{b}$ Lawrence Berkeley National Laboratory, 1 Cyclotron Road, Berkeley, CA, USA \\ 94720; 'Physics Department, University of Michigan, Ann Arbor, MI, USA 48109; \\ ${ }^{\mathrm{d}}$ Department of Physics \& Astronomy, University College London, Gower Street, London, \\ UK WC1E 6BT;
}

\begin{abstract}
The Dark Energy Spectroscopic Instrument (DESI) is a project in construction to measure the expansion history of the Universe using the Baryon Acoustic Oscillation technique. The spectra of 35 million galaxies and quasars over 14,000 square degrees will be measured during the life of the experiment. A new prime focus corrector for the KPNO Mayall telescope will deliver light to 5000 fiber optic positioners. The fibers in turn feed ten broad-band spectrographs covering a $360-980 \mathrm{~nm}$ passband with a spectral resolution $(\lambda / \Delta \lambda)$ between 1500 and 4000 . The spectrograph uses two dichroic beam splitters to separate the flux among three spectral cameras, each with a volume phase holographic grating and lens system that focuses onto a charge coupled device detector. We describe the spectrograph, its system requirements, design and construction.
\end{abstract}

Keywords: DESI, dark energy, multi-object spectrograph, Mayall telescope

\section{INTRODUCTION}

The DESI instrument ${ }^{1}$ is being developed for the KPNO Mayall telescope to measure the spectra of 35 million galaxies and quasars. A prime focus telescope corrector will deliver light to 5000 fiber optic positioners. The fibers will be distributed among ten broad-band spectrographs that cover a 360 - $980 \mathrm{~nm}$ passband with a spectral resolution $(\lambda / \Delta \lambda)$ between 1500 and 4000 . The first spectrograph has been delivered to the telescope and all ten of them are planned to be installed by May 2019.

The spectrograph requirements are shown in Table 1. Primary requirements include the bandpass, spectral resolution, throughput, point spread function (PSF) stability, and noise variance. The fiber size was chosen to optimize the signal-tonoise ratio in emission-line galaxies as seen on the sky.

More detailed spectrograph specifications are shown in Table 2. The 5,000 fibers are placed on slits of 10 spectrographs. The detectors chosen were $4 \mathrm{k}$ x $4 \mathrm{k}, 15 \mu \mathrm{m}$ pixel charge coupled device (CCD) detectors.

*jerrye@ssl.berkeley.edu; phone +1 5106420599

Ground-based and Airborne Instrumentation for Astronomy VII, edited by Christopher J. Evans, Luc Simard, Hideki Takami, Proc. of SPIE Vol. 10702, 107027G · @ 2018 SPIE · CCC code: 0277-786X/18/\$18 - doi: 10.1117/12.2311821 
Table 1: Spectrograph Requirements.

\begin{tabular}{lrr}
\hline Item & Requirement & Current Design \\
\hline Bandpass & $360-980 \mathrm{~nm}$ & complies \\
Resolution $(\lambda / \Delta \lambda)$ & $\geq 1,500 ; 360 \mathrm{~nm}<\lambda \leq 555 \mathrm{~nm}$ & $2,000-3,200$ \\
& $\geq 3,000 ; 555 \mathrm{~nm}<\lambda \leq 656 \mathrm{~nm}$ & $3,200-4,100$ \\
& $\geq 4,000 ; 656 \mathrm{~nm}<\lambda \leq 980 \mathrm{~nm}$ & $4,100-5,100$ \\
& $\geq 21 \% ; \lambda=360 \mathrm{~nm}$ & $39 \%$ \\
& $\geq 30 \% ; \lambda=375 \mathrm{~nm}$ & $51 \%$ \\
& $\geq 43 \% ; \lambda=400 \mathrm{~nm}$ & $60 \%$ \\
& $\geq 57 \% ; \lambda=500 \mathrm{~nm}$ & $69 \%$ \\
& $\geq 50 \% ; \lambda=600 \mathrm{~nm}$ & $69 \%$ \\
End-to-end throughput & $\geq 58 \% ; \lambda=700 \mathrm{~nm}$ & $70 \%$ \\
& $\geq 63 \% ; \lambda=800 \mathrm{~nm}$ & $78 \%$ \\
& $\geq 62 \% ; \lambda=900 \mathrm{~nm}$ & $73 \%$ \\
& $\geq 48 \% ; \lambda=980 \mathrm{~nm}$ & $55 \%$ \\
Point Spread Function Stability & $\leq 1 \%$ & complies \\
Number of Fibers & 5,000 & 5,000 \\
Fiber diameter & $107 \mu \mathrm{m}$ & $107 \mu \mathrm{m}$ \\
Collimator f/\# & $\leq 3.57$ & 3.57 \\
Noise Variance & $-10^{\circ} \mathrm{C}$ to $+30^{\circ} \mathrm{C}$ & complies \\
Operational Temperature & $-20^{\circ} \mathrm{C}$ to $+40^{\circ} \mathrm{C}$ & complies \\
Survival Temperature & & complies \\
\hline
\end{tabular}

Table 2: Spectrograph Baseline Design Specifications.

\begin{tabular}{lrr}
\hline Item & Specification & Current Design \\
\hline Number of spectrographs & 10 & complies \\
Detector pixel pitch & $15 \mu \mathrm{m}$ & $15 \mu \mathrm{m}$ \\
Spectral detector elements & 4,096 pixels & complies \\
Spatial detector elements & 4,096 pixels & complies \\
Minimum resolution elements & $\geq 3$ pixels & 3.4 pixels \\
Fiber spacing (slit plane) & $230 \mu \mathrm{m}$ & $230 \mu \mathrm{m}$ \\
Slit Height & $120.9 \mathrm{~mm}$ & $120.9 \mathrm{~mm}$ \\
Number of fibers (spatial) & 500 & 500 \\
Maximum rms Radius & $\leq 13 \mu \mathrm{m}$ & $11.5 \mu \mathrm{m}$ \\
Fiber - Fiber Crosstalk & $\leq 0.5 \%$ & $0.05-0.46 \%$ \\
$95 \%$ Encircled energy diameter & $\leq 110 \mu \mathrm{m}$ & $61-82 \mu \mathrm{m}$ \\
$50 \%$ Encircled energy diameter & $\leq 50 \mu \mathrm{m}$ & $37-45 \mu \mathrm{m}$ \\
Operational Temperature & $20^{\circ} \mathrm{C} \pm 2^{\circ} \mathrm{C}$ & complies \\
\hline
\end{tabular}

The schematic and rendering of the spectrograph system is shown in Figure 1. The light from the fiber slit is collimated by a spherical mirror. The light is then split into three spectral band passes using two dichroics. Each of the three channels disperses the light with a volume phase holographic (VPH) grating. The light is then focused onto the detector using five lenses in three groups. The collimator accepts the f/3.57 light output from the fibers, allowing for focal ratio degradation (FRD) after the $\mathrm{f} / 3.85$ light input. The design chosen has the maximum magnification possible that meets the resolution requirements. This results in the slowest possible camera (f/1.7), which is easier to manufacture. The bands chosen that meet the requirements are: $360-593 \mathrm{~nm}, 566-772 \mathrm{~nm}$, and 747-980 nm for the blue, red and near infrared (NIR) channels respectively. 


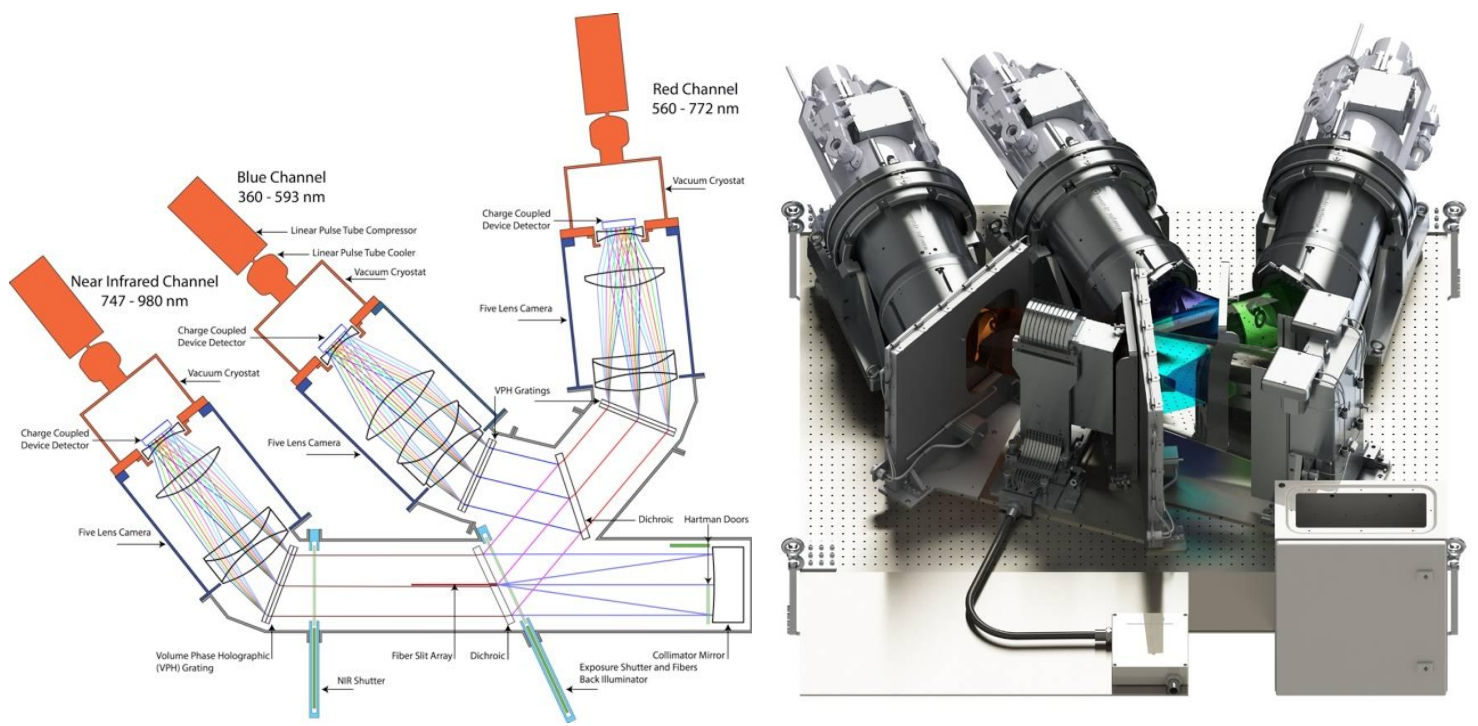

Figure 1. A schematic (left) and rendering (right) of the DESI spectrograph optical and mechanical systems. The spectrograph is $1.8 \mathrm{~m}$ wide $\times 1.4 \mathrm{~m}$ deep $\times 0.6 \mathrm{~m}$ high.

\section{THE SPECTROGRAPH COMPONENTS}

The spectrograph components are described in order of the optical path.

\subsection{Optical Components}

The slit, which is the input to the spectrograph, consists of 500 fiber end-faces arranged into a fiber slit ${ }^{2}$. The fibers are arranged in a radius of $468 \mathrm{~mm}$, near the collimator focus. The $107 \mu \mathrm{m}$ diameter fibers are mounted with $230 \mu \mathrm{m}$ separation in groups of 25 in v-groove blocks. Adjacent v-groove blocks are spaced by $556 \mu \mathrm{m}$, providing an image gap to measure the PSF wings in the spectral extraction process. The total fiber slit is $120.9 \mathrm{~mm}$ length of the arc including the first and last fibers. The slit is inserted into a slot milled into the first dichroic so that the tip of the center fiber is coincident with the dichroic reflective surface.

The fiber's output illuminates the collimator. We chose a reflective versus a refractive collimator given its fewer optical surfaces (only one) and improved throughput. The spherical collimator mirror, made by Winlight Optics, operates at $\mathrm{f} / 3.57$ and produces a $126 \mathrm{~mm}$ pupil. A dielectric enhanced silver coating by Infinite Optics was chosen for its higher reflectance in the DESI bandpass, shown Figure 2. Winlight has polished all 10 collimators and 10 have been coated by Infinite Optics.

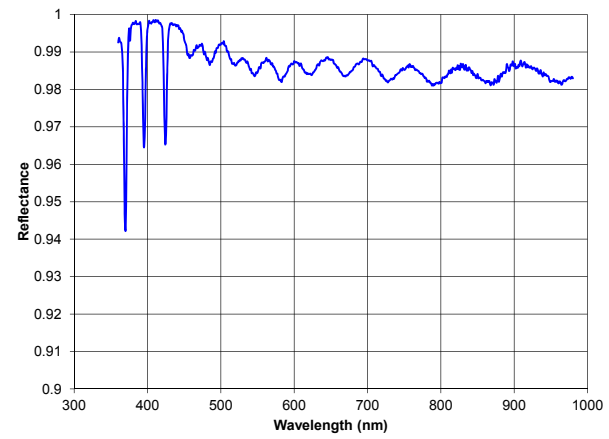

Figure 2: The reflectance of the enhanced DESI silver coating for the collimator. The blue curve is a DESI measurement from the first collimator.

The total bandpass is divided into three channels using two dichroic beam splitters. The dichroics are located after the collimator in the parallel beam. The first dichroic transmits the NIR band and then second dichroic transmits the red 
band. The filters are fused silica with the dichroic coatings on one side and combined anti-reflection and stress compensation layers on the other. The dichroic filters specifications are listed in Table 3. All 20 dichroics were made by Materion Barr. The throughput measurements are shown in Figure 3.

Table 3: Dichroics Budgeted Requirements.

\begin{tabular}{lccl}
\hline & NIR Pass Dichroic & Red Pass Dichroic & Throughput \\
\hline Reflection Band (nm) & $360-747$ & $360-566$ & $>95 \%$ \\
Transmission Band (nm) & $772-980$ & $593-747$ & $>95 \%$ \\
Crossover Region R+T (nm) & $747-772$ & $566-593$ & $>90 \%$ \\
Crossover Width (nm) & 25 & 27 & \\
\hline
\end{tabular}

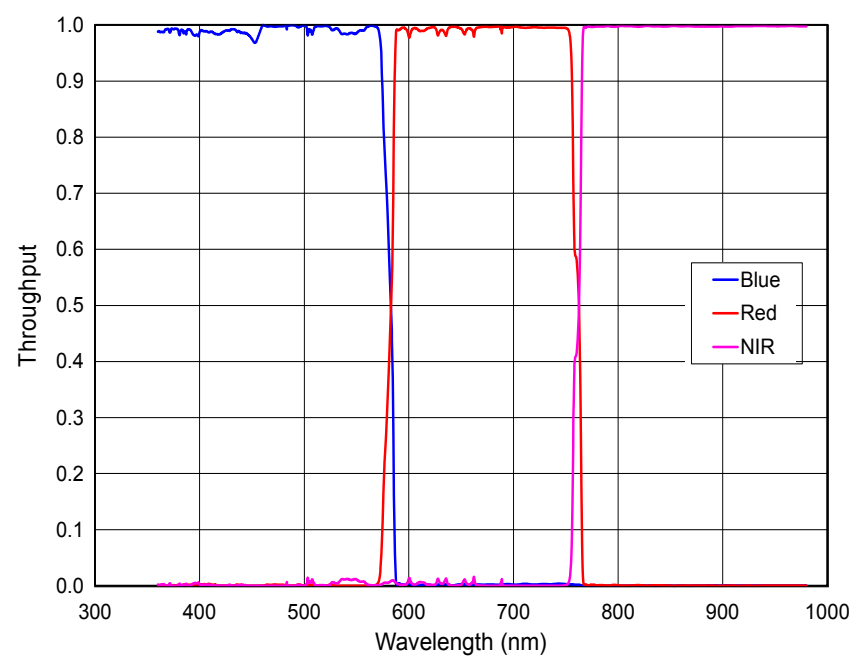

Figure 3: The measured throughput for the first set of DESI dichroics.

The camera band passes and grating parameters are given in Table 4. Volume phase holographic (VPH) gratings have been chosen as the spectral dispersers because this type of grating delivers high throughput over the relatively broad band passes of the three channels. Fringes are holographically recorded in the gelatin. In Table $4, \Delta \beta$ is the diffracted angular range, $\alpha$ is the incident angle, $1 / \sigma$ is the line density, and $\varphi$ is the tilt angle of the fringes. The fringes of the gratings are tilted to remove the Littrow ghost ${ }^{3}$. Our studies showed that high efficiency is maintained at the band pass edges when the grating diffracted angular range $(\Delta \beta)$ was kept to less than $16^{\circ}$. The clear aperture of the gratings is kept below $145 \mathrm{~mm}$ so that production holographic recording tables can be used to reduce the cost.

Table 4: Spectrograph Grating Budgeted Requirements.

\begin{tabular}{lcccl}
\hline & Blue Channel & Red Channel & NIR Channel & Driver \\
\hline$\lambda_{\min }(\mathrm{nm})$ & 360 & 566 & 747 & \\
$\lambda_{\max }(\mathrm{nm})$ & 593 & 772 & 980 & \\
$\Delta \beta\left(^{\circ}\right)$ & 15.74 & 15.5 & 15.42 & Throughput \\
$\alpha\left(^{\circ}\right)$ & 10.44 & 18.12 & 20.76 & Littrow ghosts \\
$1 / \sigma(l / m m)$ & 1101.9 & 1157.4 & 992.5 & Littrow ghosts \\
$\phi\left(^{\circ}\right)$ & -3.18 & -3.03 & -2.97 & Littrow ghosts \\
Band Edge Efficiency & $>65 \%$ & $>80 \%$ & $>80 \%$ & Throughput \\
\hline
\end{tabular}

All thirty gratings were made by Kaiser Optical Systems (KOSI) and tested for efficiency and wave front error by Lawrence Berkeley National Laboratory (LBNL). Figure 4 shows a photo of the three gratings in a test mount. Figure 5 shows measurements of the wave front error, taken with a Zygo interferometer. The peak to valley wave front error for 
power and irregularity are below the budgeted 1.5 and 1.0 waves respectively. The average efficiency of each type of grating is shown in Figure 6. The efficiency is optimized by adjusting the incidence angle, within an allowed installation range of $< \pm 1^{\circ}$.

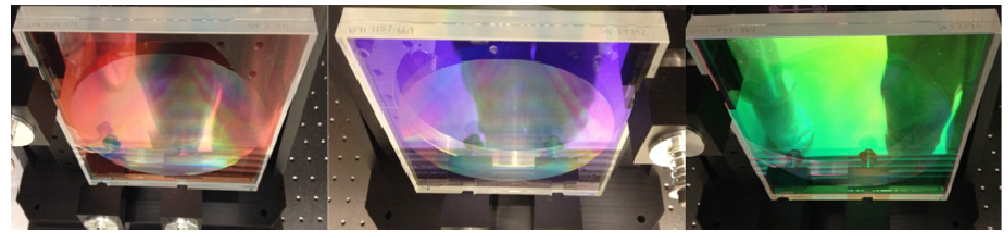

Figure 4: Photographs of the blue, red and NIR gratings in test mounts, from left to right.

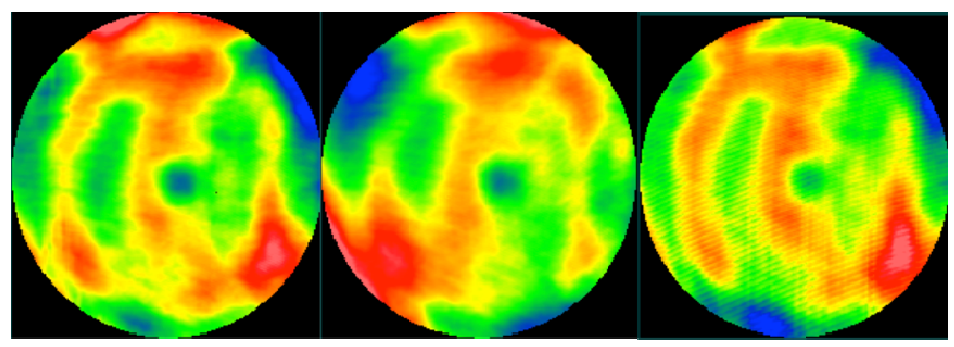

Blue: $P$ Wr $=0.04$, Irr $=0.82 \quad$ Red: $P w r=0.40,1 \mathrm{Ir}=0.97 \quad$ IR: $P$ Wr $=0.63, \mathrm{Irr}=0.80$

Figure 5: Measured blue, red and NIR grating wave front in -1 order, from left to right. Power and Irregularity wave front are indicated by Pwr and Irr, respectively.

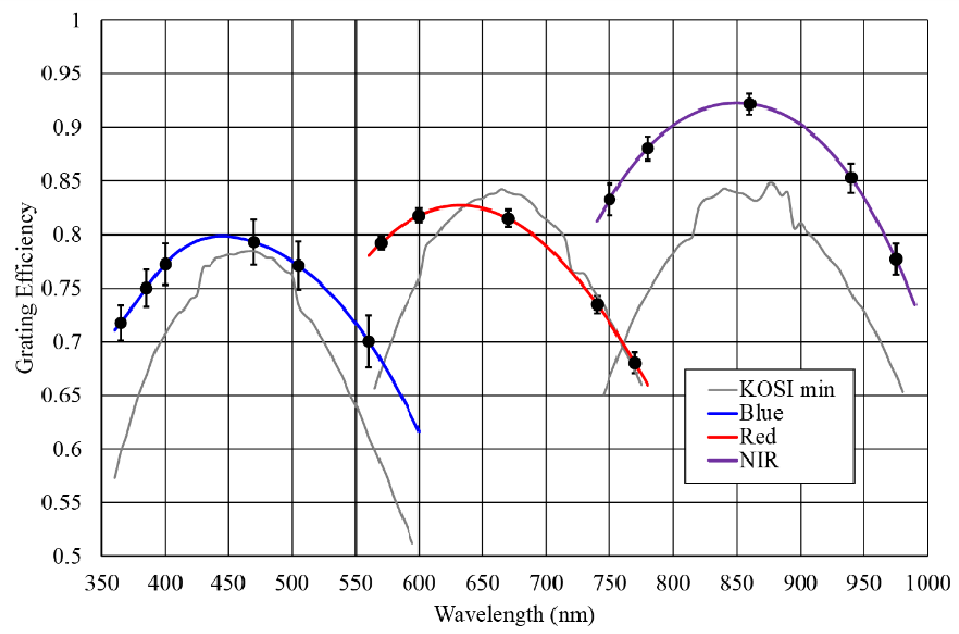

Figure 6: The LBNL measured average area-weighted grating efficiencies and their uncertainties are plotted for each passband. The corresponding average spline interpolations are shown as colored curves. The KOSI minimum efficiency specifications are overplotted as grey curves. Although the measured Red curve falls below the expected minimum, the performance is acceptable as the science yield is mitigated by the total efficiency over the band.

\subsection{Spectrograph Cameras}

The spectrograph design space was studied to consider whether the optics should be refractive or reflective. Refractive cameras were chosen for the trades of throughput, imaging quality, and complexity and costs. Refractive cameras have no obscuration but require more optical surfaces. Reflective Schmidt cameras use an aspheric corrector plate, spherical mirror and field flattener lenses and the detector obscures the beam with a loss of at least $10-15 \%$. 
Since the spectral range is more than a factor of two, the spectrograph bandpass needs to be split into at least two channels, using dichroic filters, to eliminate second order contamination. Three channels were chosen for two reasons: the grating efficiency is increased since they cover less bandpass each, and using less than 3 channels would require larger detectors than standard 4096 x 4096 pixel detectors.

The focal ratio of the camera will determine the spectral resolution of the camera and the focal length will determine the spectral coverage. The optical requirements for the cameras are given in Table 5. An area of the detector less than the full size was used to image the spectra in order to reduce the impact of edge effects and allow for alignment tolerances. The throughput requirements are budgeted from the requirements in Table 1. Camera design considerations to reduce cost included: minimize the number of lenses, minimize the number of aspheric surfaces, minimize the volume of glass, and use low cost glass with frequent melts. To maximize throughput, the number of lenses was minimized and only glasses with high transmission in the band pass were used. The last lens is fused silica since it is the vacuum seal to the cryostat. Fused silica is very strong and stiff and it is not radioactive which would increase the backgrounds in the detector.

Table 5: Camera Optical Budgeted Requirements.

\begin{tabular}{|c|c|c|c|}
\hline & Blue & Red & NIR \\
\hline Maximum rms spot radius $(\mu \mathrm{m})$ & $<12$ & $<12$ & $<12$ \\
\hline Angular field $\left(^{\circ}\right)$ & 7.72 & 7.72 & 7.72 \\
\hline Spectral field (nm) & $360-593$ & $566-772$ & $747-980$ \\
\hline Spectral field size $\left({ }^{\circ}\right)$ & 8.06 & 8.03 & 8.03 \\
\hline Focal length (mm) & $212.8 \pm 1$ & $213.6 \pm 1$ & $214.0 \pm 1$ \\
\hline Focal Ratio & $\mathrm{f} / 1.70$ & f/1.70 & $\mathrm{f} / 1.70$ \\
\hline Spectral Detector Size (mm) & 60.26 & 60.26 & 60.26 \\
\hline Spatial Detector Size (mm) & 60.02 & 60.02 & 60.02 \\
\hline \multirow{5}{*}{ Throughput } & $>86 \%$ & $>92.5 \%$ & $>92.4 \%$ \\
\hline & @ $\lambda=360 \mathrm{~nm}$ & $@ \lambda=560 \mathrm{~nm}$ & @ $\lambda=740 \mathrm{~nm}$ \\
\hline & $>90 \%$ & $>92 \%$ & $>91 \%$ \\
\hline & @ $\lambda=600 \mathrm{~nm}$ & @ $\lambda=650 \mathrm{~nm}$ & @ $\lambda=980 \mathrm{~nm}$ \\
\hline & & $\begin{array}{c}>93 \% \\
@ \lambda=780 \mathrm{~nm}\end{array}$ & \\
\hline
\end{tabular}

The optical design was only allowed to have a maximum RMS radius of $<10 \mu \mathrm{m}$ to allow room for manufacturing and alignment tolerances. All of the channels have 5 lenses with 2 aspheric surfaces (the front of the first lens and the front of the last lens). The detector cryostat window and CCD are tilted and offset from the optical axis. The blue camera uses three glass types including CaF2, N-BAK2 and fused silica. The red and NIR cameras have 4 standard glass types, BSM4, TIM22, LAL12 and fused silica. A schematic of the cameras is included in Figure 1. The spot sizes for the spectrograph are shown in Figure 7. The maximum RMS radius versus wavelength is in Figure 8. The design meets the requirement of $<10 \mu \mathrm{m}$ RMS at all wavelengths.

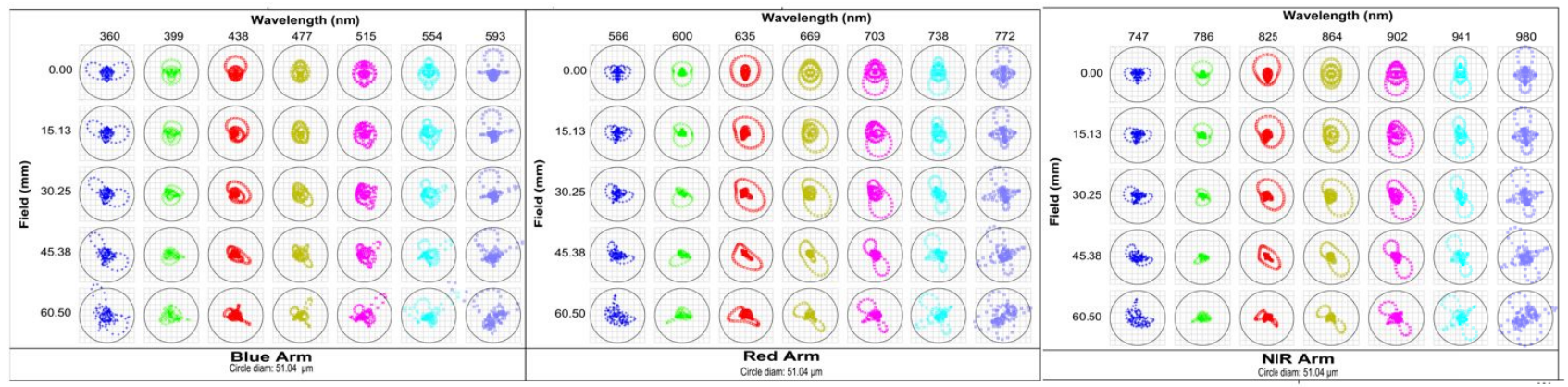

Figure 7: The ray traced spot diagram for the spectrograph. The left figure is for the blue channel, the middle figure is for the red channel and the right figure is for the NIR channel. For each figure different fiber locations are shown from top to bottom (from 0.0 to $60.5 \mathrm{~mm}$ ) and different wavelengths are shown from left to right. The circle is the size of the projected fiber onto the detector. 


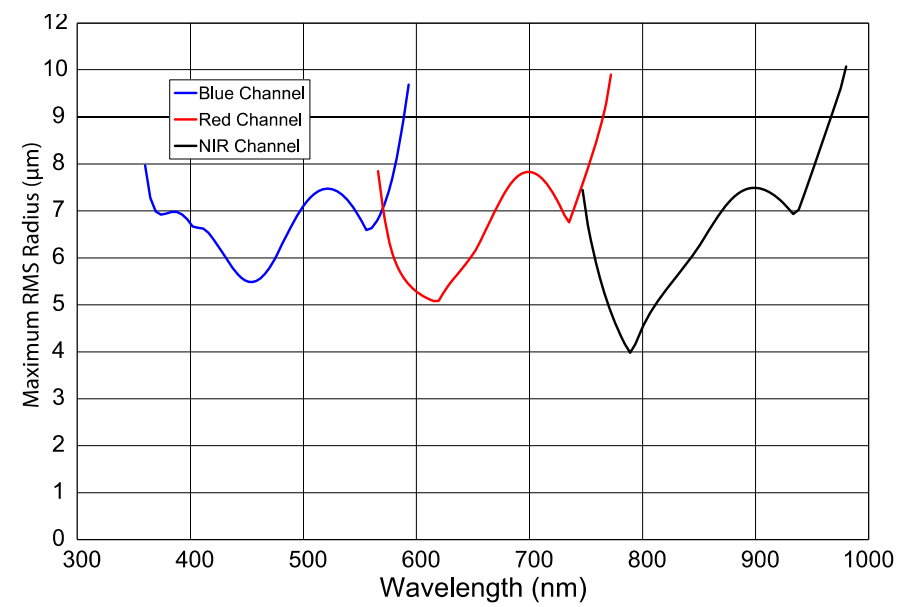

Figure 8: The maximum RMS radius versus wavelength for the optical design is plotted. The blue, red and black curves are for the blue, red and NIR channels of the spectrograph respectively. All wavelengths meet the $10 \mu \mathrm{m}$ RMS requirement for the optical design.

An optical thermal analysis of the cameras was done for the operation range $18-22^{\circ} \mathrm{C}$. The red and the NIR cameras did not require passive athermalization. The blue camera incorporates passive athermalization by using a plastic with a compensating thermal expansion coefficient to locate the fourth lens cell.

The red and NIR triplets are bonded with UV curing glue. An FEA analysis of the triplet was done to determine that the stress and strain on the glass and the glue would not cause the triplet to fail. The first red triplet was validated for three thermal cycles for the DESI survival temperature range of -20 to $+40{ }^{\circ} \mathrm{C}$. A test bonded blue triplet failed survival temperature tests so it was redesigned to use an oil coupling scheme following the design used by the KOSMOS spectrograph $^{5}$. Cargille 1074 laser liquid was chosen for the fluid. The transmission did not change when the oil was used with silicone o-rings and diaphragm materials for a 30-month life test. The oil cell was also validated with thermal cycle testing.

The mechanical design of the blue camera is shown in Figure 9. All the camera elements (see Figure 10) and assemblies have been fabricated, aligned and tested by Winlight Systems, including a full set which has been integrated into the first DESI spectrograph system.

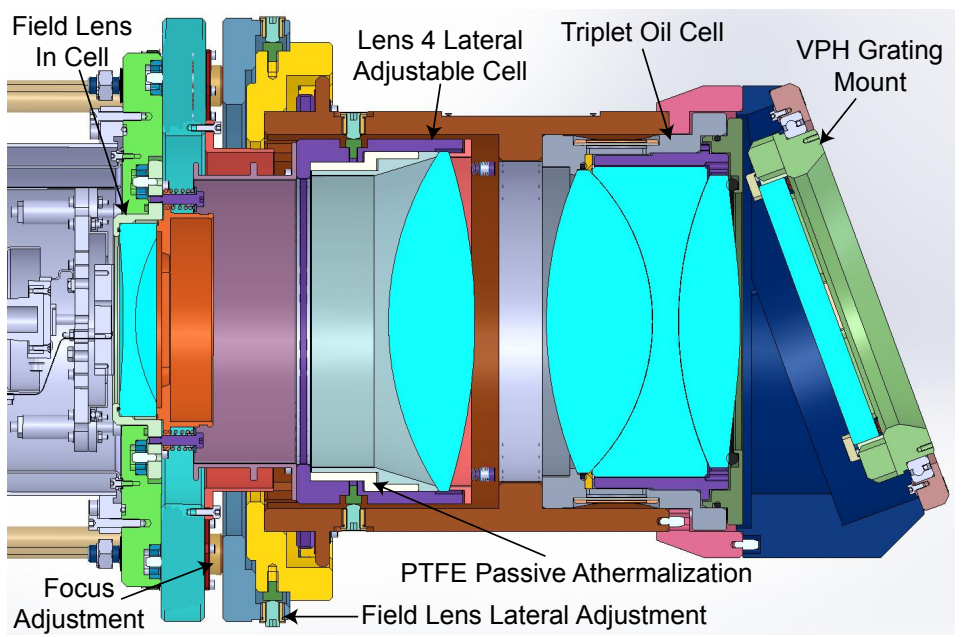

Figure 9: Section of the mechanical design of the blue camera. The VPH grating mount is shown in dark blue on the right. The triplet oil cell is shown next in gray and purple. The lateral adjustable cell for the fourth lens is shown next in pink and purple. Finally, the field lens cell is shown in light green with an orange base. All of the lens cells are thermally compensated axially and radially. The L4 cell is athermalized using a PTFE compensation piece in white. 

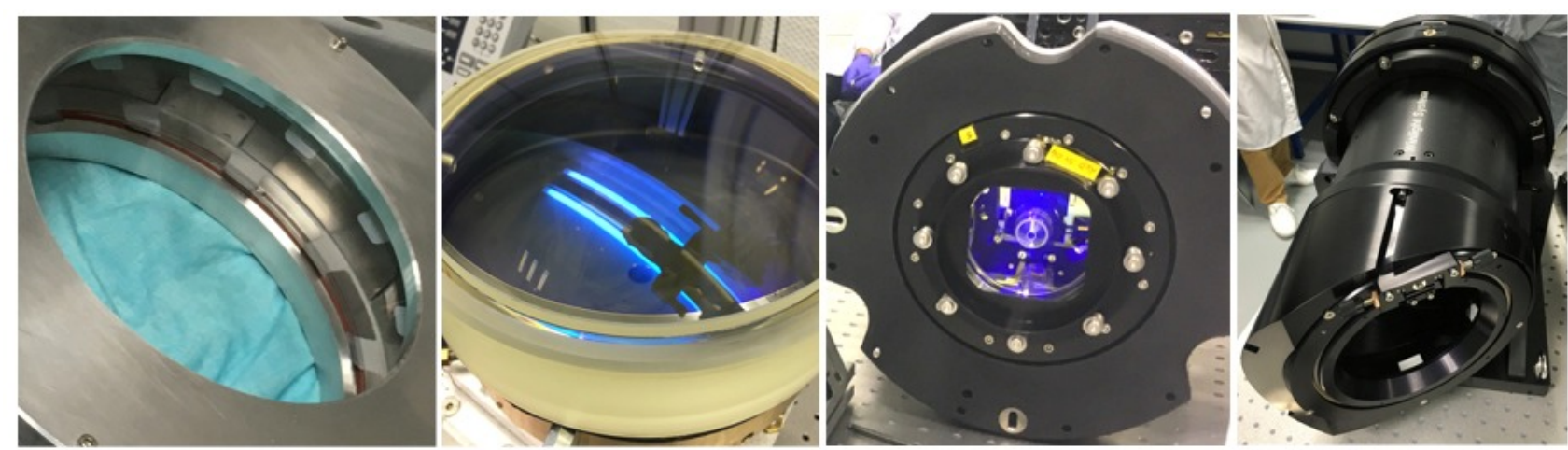

Figure 10: Cameras components under development (left to right): The demonstration test oil cell for the blue camera. The cemented $200 \mathrm{~mm}$ diameter triplet for the red camera. The red camera field lens assembly mounted in its cryostat interface is shown with a focal plane microscope behind it. A camera barrel mechanical assembly.

\subsection{Camera CCD Cryostats}

The ten DESI spectrographs each require three CCDs for the blue, red, and NIR channels. Each CCD is housed in its own cryostat that has its own refrigerator and CCD control electronics. The cryostat, developed by the CEA Saclay, France, provides the mechanical connection to the spectrograph camera, supports the thermal and vacuum conditions, and interfaces with the control system and the CCD electronics. The cryostat is rendered in cross section in Figure 11 and a working cryostat system is shown in Figure 12.

The system cools the CCDs to $140 \mathrm{~K}$ (red and NIR channels) or $163 \mathrm{~K}$ (blue channel) with a precision of $1 \mathrm{~K}$ and regulate their temperature to $\pm 0.1 \mathrm{~K}$. The cryostats are identical except for the entrance vacuum window which also serves as the last camera optical element. The reference and mounting plane between the spectrograph and the CCD is the front face of the cryostat flange. The CCD's must be aligned in transverse and tilt axes relative to the spectrograph optical axis. The CCD plane is positioned with shims and is fixed to the cold plate with bolts that can align the CCD plane within 100-200 $\mu \mathrm{m}$ along the optical $\mathrm{Z}$ axis, and within $50 \mu \mathrm{m}$ in transverse $\mathrm{XY}$ plane. The CCD columns are aligned within 3 arcminutes relative to the dispersion direction. The final CCD focusing precision, $\pm 15 \mu \mathrm{m}$ along $\mathrm{Z}$, is achieved by moving the camera relative to the cryostat by adjusting screws.

Cooling power is supplied to the CCD on its Invar package via an Invar cold plate connected with flexible copper braids to a copper cold base screwed to the cold tip of the cryocooler. Thermal sensing resistors fixed on the CCD package, the cold plate, the cold tip and on the front flange close to the field lens assembly to ensure thermal control and monitoring. Thermal shielding using polished Al plates is provided for the vessel sides, the rear flange and one for the front lens.

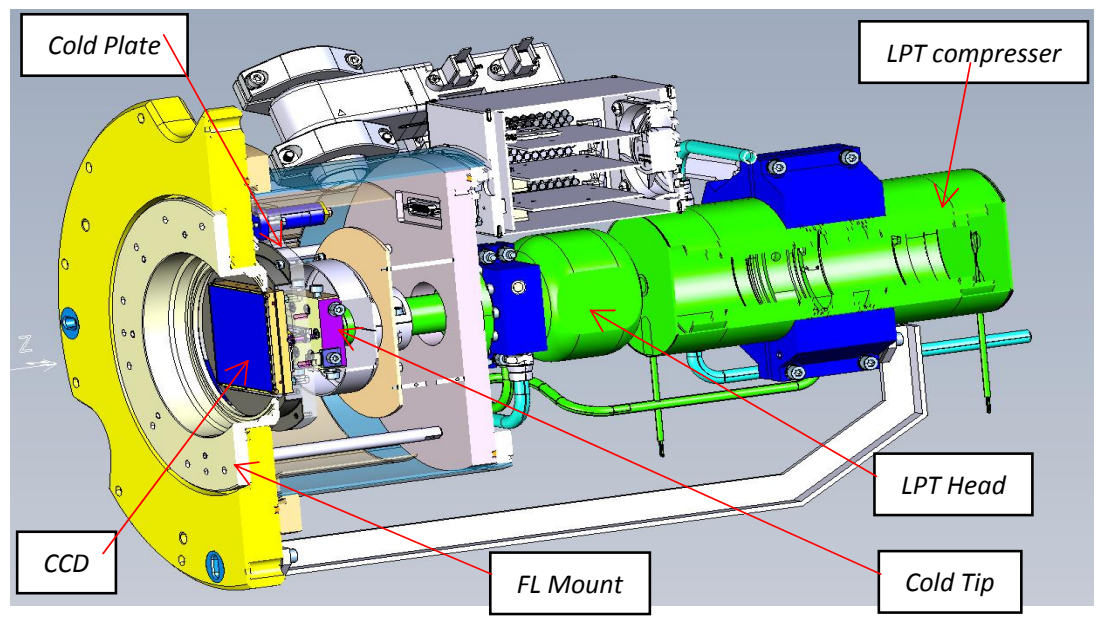

Figure 11: A model of the cryostat and camera interface. A cryostat weighs $30 \mathrm{~kg}$, is $310 \mathrm{~mm}$ in diameter and $480 \mathrm{~mm}$ long. 
Each cryostat uses a closed cycle pulse tube cooler (Thales LPT9310 Cryocooler with a thermal power of 4.5 to $5.6 \mathrm{~W}$ at $80 \mathrm{~K}$ ). Manufactured cryostats demonstrated good performance. The measured thermal losses were $2.8 \mathrm{~W}$ to $3.2 \mathrm{~W}$. The CCD temperature precision was $<1{ }^{\circ} \mathrm{K}$ and the temperature stability was $\pm 0.1{ }^{\circ} \mathrm{K}$. The cooler worked at $25-40 \%$ of its maximal power, while being cooled with Glycol at $10{ }^{\circ} \mathrm{C}$. The cool down temperature rate was $1.5^{\circ} \mathrm{K} / \mathrm{min}$ and temperature stability was achieved within 4.5 to 6 hours for all the tested cryostats.
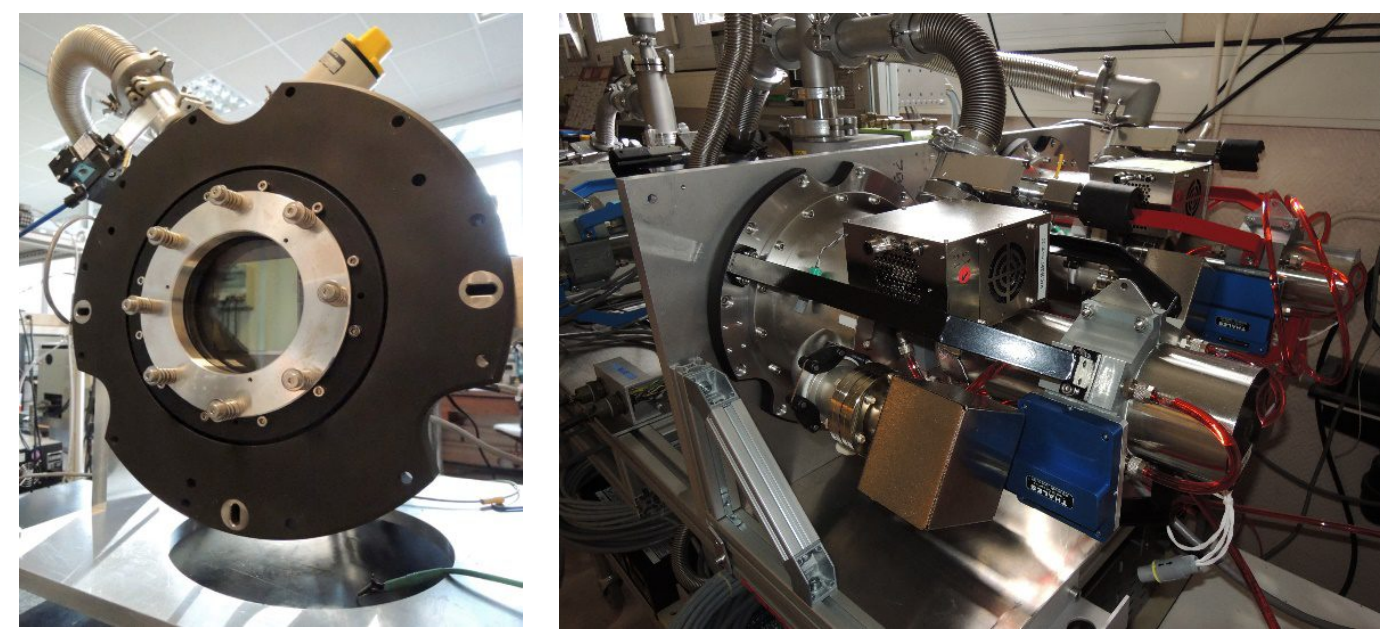

Figure 12: An operational cryostat assembly is shown on the left with a view showing a flat test field lens assembly. The cryostat structure including the cryocooler, its controller, and the CCD front electronics box is shown on the right.

The cooler compressor pistons are driven by integral linear electric motors and are gas-coupled to the pulse tube cold finger. Isolation and damping of the compressor has been tuned as has the frequency of each cooler so that the vibrations of the 30 coolers do not add in phase. Vibration amplitude was less than $3 \mu \mathrm{m}$ at the detector. A remaining tiny amplitude vibration modulation ( $1 \mu \mathrm{m}$ over 6.5 hours) was detected on test images. That was generated by a difference of $40 \mu \mathrm{Hz}$ between the $47 \mathrm{~Hz}$ of the 3 coolers. The coolers difference frequencies were set at $1 \mathrm{~Hz}$ to smear out the modulation for exposures longer than 1 second.

A cryostat control system made by CEA Saclay, France, has been built to independently drive and maintain all 30 channels, and communicates with the DESI Instrument Control System ${ }^{6}$. The cryostats' vacuum will be maintained by an ion pump. Supervision software controls the cryocoolers and the vacuum system and allows the set-up to be remotely controlled. The total electrical consumption for the cryostat system is $11 \mathrm{~kW}$ during cool-down of the 30 units and reduced to 3 to $4 \mathrm{~kW}$ in nominal operation condition. 


\subsection{Sensors and Electronics}

The spectrograph cameras each use a $4 \mathrm{k} \times 4 \mathrm{k} C \mathrm{CD}$ with $15 \mu \mathrm{m}$ pixels. A subset of performance specifications is given in Table 6 . For the blue channel we baseline 10 of the ITL STA4150A CCDs provided by University of Arizona Imaging Technologies Lab. For the red and NIR channels we baseline 20 of the LBNL $4 \mathrm{k} \times 4 \mathrm{k}, 250 \mu \mathrm{m}$ thick CCDs to optimize quantum efficiency (QE). The sensors are shown in Figure 13. Currently 21 LBNL and 8 ITL detectors are fabricated and meet the requirements. The blue curve in Figure 14 shows the QE of the ITL CCD. The red curve in Figure 14 shows measured QE for an LBNL CCD with a broadband antireflection coating. The high-side cutoff is determined by the CCD thickness.

Table 6: CCD performance specifications

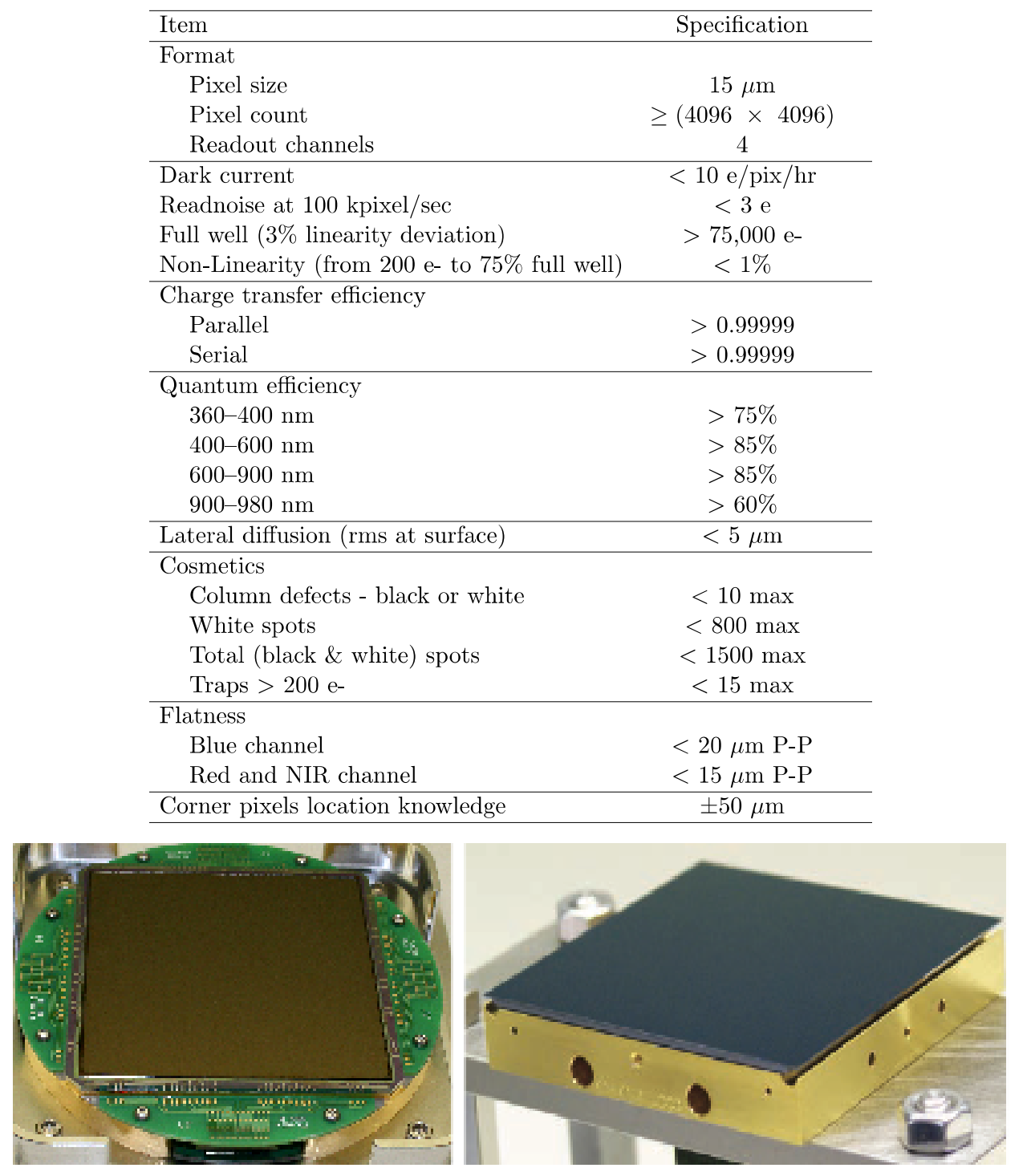

Figure 13: The packaging scheme for LBNL and ITL CCDs that are compatible with the DESI cryostats are shown. CCDs are 4k x 4k with $15 \mu \mathrm{m}$ pixels. Left: ITL package for STA 4150A CCD. Right: LBNL 4k x 4k CCD package. 


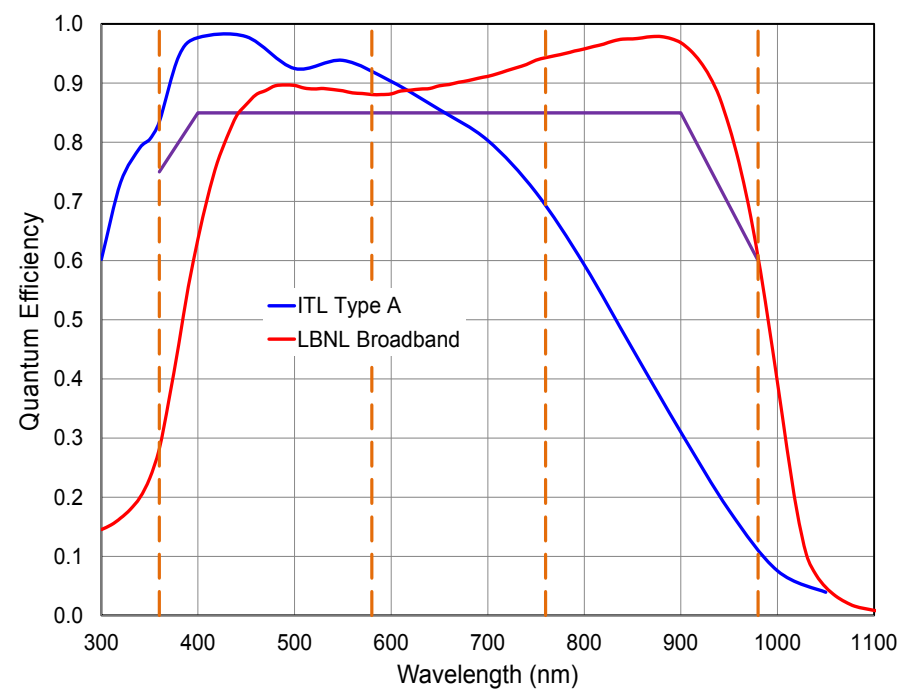

Figure 14: Quantum e ciency for DESI: ITL STA5140A and $250 \mu \mathrm{m}$ thick LBNL CCDs. Data are lab measurements. The purple line is the CCD specification. The vertical orange dashed lines are the locations of the band transitions for the three spectrograph channels. The ITL sensor, in the Blue band, and LBNL sensor, for the Red and NIR bands, meets their specifications.

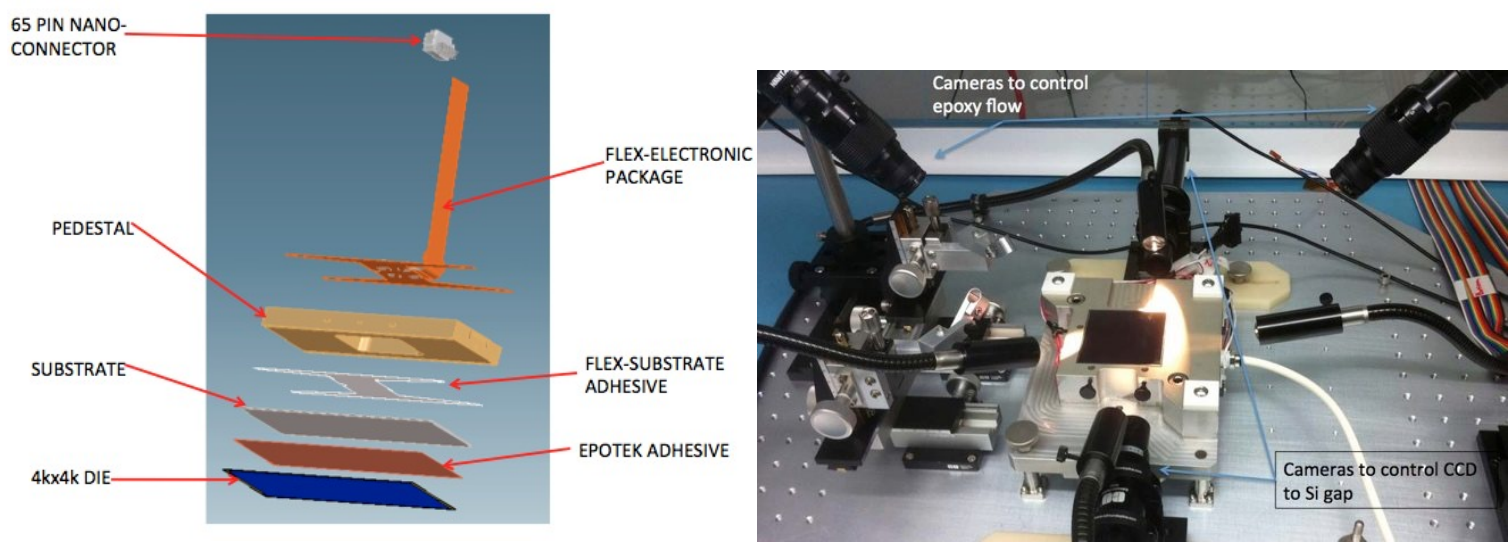

Figure 15: Left: Blowup of CCD package for the DESI 4k x 4k LBNL CCDs. Right: CCD packaging fixture showing four digital cameras to control the process. Two are used to measure the gap between the CCD and the substrate, which can be adjusted with micrometers (not shown in the image). The other two cameras are used to monitor the flow of epoxy during the gluing process. The fixture holding the substrate is not shown in this image.

ITL will provide packaged and tested CCDs. LBNL will produce and cold probe unpackaged CCD die that will in turn be packaged and characterized at FNAL. An exploded view of the package for the 4k x 4k DESI sensors is shown in left of Figure 15. The detector is attached to a Si substrate with Epotek adhesive. The Si substrate is attached to the custom readout flex circuit, which brings the signals to a 65-pin connector. The flex circuit is wire-bonded to the CCD. Finally, an Invar pedestal foot is attached to the substrate to provide mechanical support for the detector. The $80 \mu \mathrm{m}$ gap and flatness between the sensor and the substrate is controlled with a measuring microscope system shown on the right of Figure 15, right. Detectors have been assembled and room temperature and cryogenic flatness measurements have been performed. An example is shown in Figure 16 where the maximum deviation from a flat surface is $6 \mu \mathrm{m}$. 

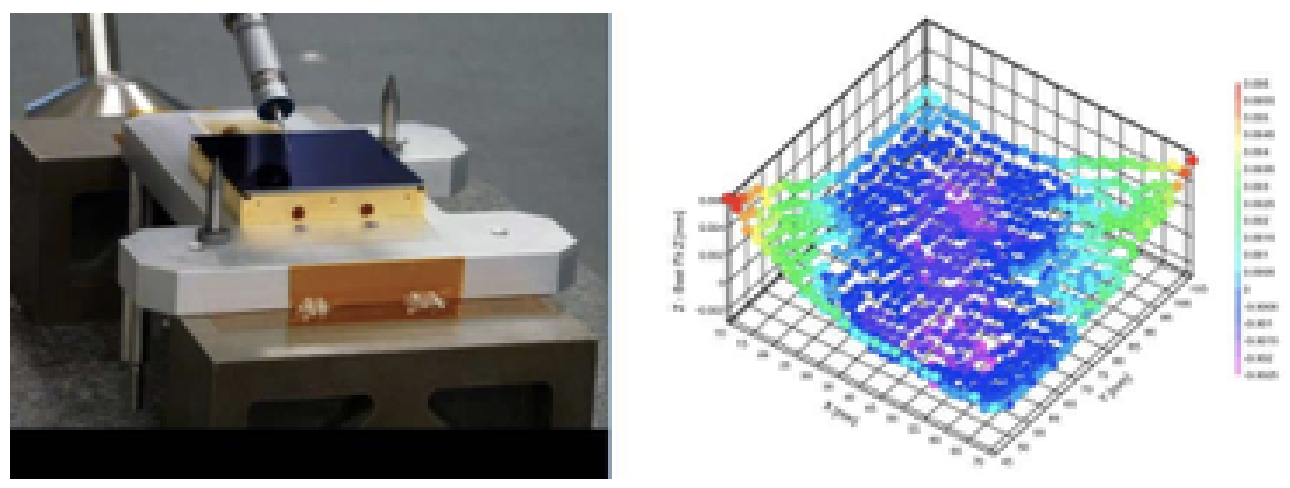

Figure 16: Left: CMM touch probe measurements of the package flatness for a completed mechanical grade device. Right: Deviations from the best fit plane on the mechanical grade device.

Detectors are fully characterized at FNAL including noise measurements, linearity measurements for the complete dynamic range of the instrument, QE measurements in the range between $360 \mathrm{~nm}$ and $1000 \mathrm{~nm}$, and dark current. The CCDs will be exposed to x-rays to characterize the di usion of the detector and charge transfer e ciency. The characterization will also include scanning clock and bias voltages around the nominal operating parameters. CCD's are tested with stations, one that uses an optical bench with a stabilized illumination source, coupled to a monochromator with a wavelength range of $360 \mathrm{~nm}$ to $1000 \mathrm{~nm}$ and operates with a stable temperature from room temperature to $145 \mathrm{~K}$. Another test station measures the flatness of detectors at the operating temperature of $145 \mathrm{~K}$. This measurement is done using a confocal lamp focusing light on the cold CCD surface, and capable of determining the reflecting plane with a precision of $1 \mu \mathrm{m}$. This light source is mounted on a precision X-Y stage to produce a topological map of the detector.

LBNL has developed a generic four-channel CCD readout controller that supports both flavors of CCD. The readout electronics module for each CCD is mounted on the warm side of the cryostat wall. The modules include local power generation from an isolated single DC voltage, CCD bias voltages generation, programmable clock levels and pattern, CCD signal processing and digitization, and operating settings readback. The module runs Linux supporting a suite of remotely callable routines for normal and special CCD operations. Configuration and control of the electronics and delivery of science data is over Ethernet. All CCDs have been operated with this system and meet their performance requirements.

\subsection{Spectrograph Mechanical Components}

The spectrograph assembly, made by Winlight Systems, and its major mechanical components are shown in Figure 1, and includes the optical bench, cameras and cryostats, optical elements, the fiber illuminator, and mechanical elements such as the shutters and the Hartmann doors.

The optical bench is an aluminum/stainless steel structure with interfaces to locate the seven opto-mechanical subassemblies: the fiber slit head, the collimator, the two dichroics, and the three camera/grating assemblies. At all of these opto-mechanical interfaces, precision-machined reference datum and kinematic-locating features are employed to ensure accurate, repeatable alignment of the optical system. The optical components contain passive thermal compensators to maintain the focus over the $18-22^{\circ} \mathrm{C}$ operational temperature range. There is an enclosure that also mounts to the bench which blocks external light and is semi-hermetic so that a purge of dry air will prevent condensation on the cold cryostat window.

The shutters, made by the Ohio State University (OSU) and shown in Figure 17, are used to block light from the CCDs during readout, including light from a fiber illuminator, described below. The exposure shutter is mounted in front of the first dichroic. The NIR shutter is mounted in front of the NIR camera. The exposure shutter is used to control the exposure of the three channels simultaneously, while the NIR shutter is needed to block the light from the fiber illuminator. Both shutters use an inflatable seal to limit light leakage. The exposure shutter is located near the pupil to ensure that all of the fibers in all channels will have the same exposure independent of the motion of the shutter. The exposure and NIR shutters open or close in $<0.5$ seconds and have demonstrated a lifetime of at $>155,000$ cycles (twice the expected number of exposures). The shutters use a direct drive stepper motor with a rack and pinion gear, the blade slides on bushings, and the inductive proximity sensors detect when the shutter is fully open and fully closed. 
The fiber illuminator, a light source that is moved in front of the fiber slit head, is used to back illuminate the fiber tips at the corrector focal plane so the DESI Fiber View Camera can measure the location of the fibers. The fiber illuminator uses a linear LED source covered with a thin film diffuser and filter. The source is placed behind a narrow slit that will direct light into the $\mathrm{f} / 2.2$ acceptance cone of each fiber end. The emitted light is limited to a 400-557 nm bandpass by a 3-mm thick Hoya B-390 filter, so that the light is detectable by the fiber view camera but does not interfere with the bandpass of the telescope's guiding system.

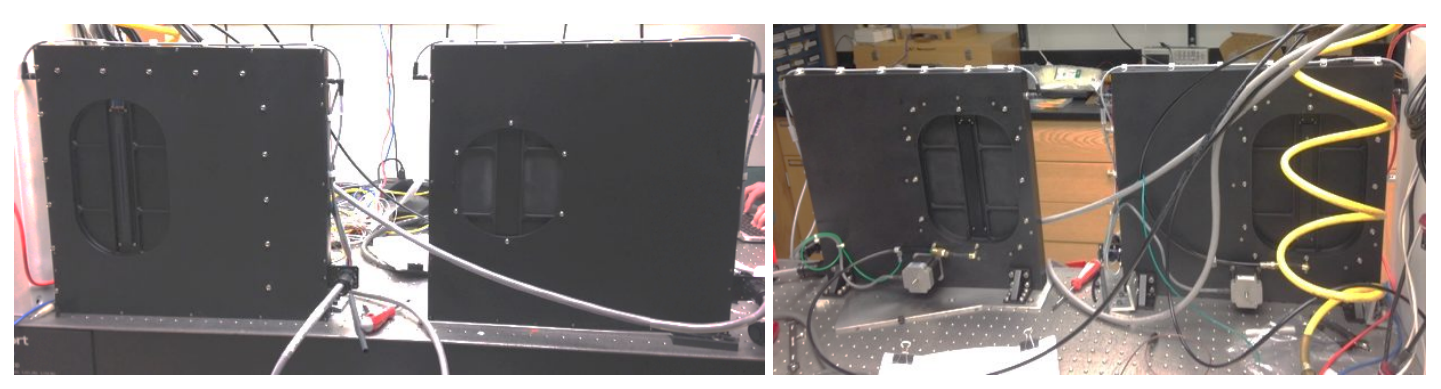

Figure 17: The shutter assemblies shown in the closed position with the exposure shutter on the left and the NIR shutter on the right. The views show the front (left) and reverse (right) sides of the shutters. The fiber illuminator LED is visible in the exposure shutter door front view. The motor and inflatable seal mechanism is visible in the reverse view.

The collimator mount, made by OSU and shown in Figure 18, has manual focus, tip, and tilt adjustments. The collimator mirror is supported with three bonded stainless-steel webs to correct for the CTE differences between the aluminum mounting plate and the fused silica mirror, yet minimize mirror distortion. An FEA indicates that the mechanical mount produces less than $5 \mathrm{~nm}$ RMS surface error in representative $128 \mathrm{~mm}$ diameter sub pupils. The collimator is athermalized so that the distance between the slit and collimator changes by less than $7.5 \mu \mathrm{m} /{ }^{\circ} \mathrm{C}$. The Hartmann doors, mounted before the collimator in its mount, are used to determine the focus by comparing the location of the centroid of spectral lines with the left or right door closed.

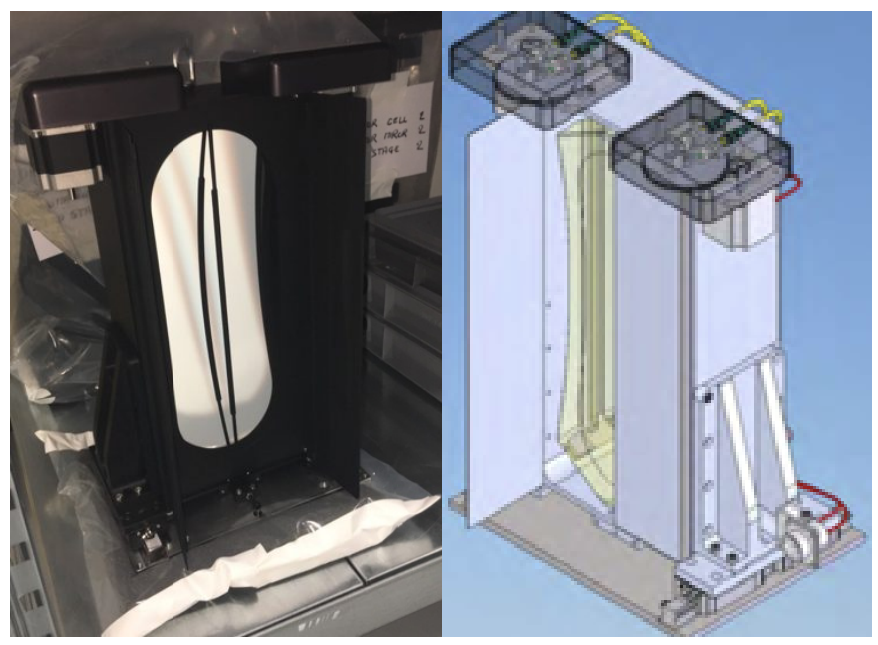

Figure 18: Left: The collimator in its mount with a fiber-slit mask and with the Hartmann doors open. Right: A rendering of the collimator mount assembly with one Hartmann door closed.

The dichroic mounts, made by OSU and shown in Figure 19, have manual tip and tilt adjustments. The NIR pass dichroic substrate has a machined slot where the slit projects toward the collimator mirror. 

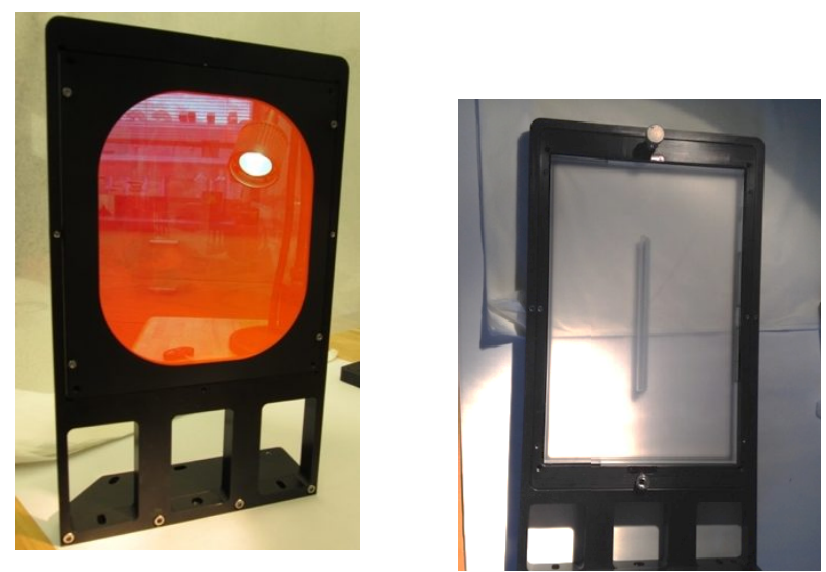

Figure 19: The mounts for the two dichroics. The one on the right has an uncoated substrate with a machined slot for fiber optic slit head to project light onto the collimator mirror.

A spectrograph controller, made by OSU, is mounted to the optical bench. The controller includes motor controllers for the shutters and Hartmann doors. It also has commands and monitors for the fiber illuminator, air valves for the shutter seals, and temperature and humidity sensors. The humidity sensors are near each cryostat window to ensure that the air is above the dew point. The spectrograph controller contains a minicomputer that operates the spectrograph, and also a router that isolates internal communication and is connected to the DESI Instrument Control System ${ }^{6}$ via Ethernet.

\section{INTEGRATED SPECTROGRAPH}

\subsection{Spectrograph Optical Tolerances and Simulation}

There are several effects that will change the imaging of the spectrograph from the optical design. The optics will not be manufactured or assembled perfectly. The illumination pattern from the fiber is not uniform. The detector will blur the images due to charge diffusion and depth of conversion. And diffraction will add wings to the images. These effects were simulated to see if the as manufactured spectrograph (including detector) will meet the imaging requirements of the spectrograph.

A Monte Carlo simulation of 1,000 spectrographs was performed to determine if the tolerances meet the requirements. The results for the red channel are shown in Figure 20. All the channels were similar to this. The most likely spectrograph had a maximum RMS radius of $<10 \mu \mathrm{m}$ and $95 \%$ of the spectrographs were better than $11 \mu \mathrm{m}$. A representative description of the as built optics was chosen from the 1,000 Monte Carlos such that there is a $95 \%$ chance that the as built camera would be better.

A measured angular illumination pattern from a fiber was used as the input into the simulated as-built optics. Diffraction was then added to the output. This combined image was then input into a detector simulator to add the charge diffusion and depth of conversion effects. Two detector types were used in the simulation. For the blue channel a thin substrate detector with $5 \mu \mathrm{m}$ diffusion was used. For the red and NIR channels a $250 \mu \mathrm{m}$ thick fully depleted model was used with a $5 \mu \mathrm{m}$ diffusion.

The FWHM spectral resolution for the simulated spectrograph is shown in Figure 21. The red curve is the minimum spectral resolution for all fibers and the blue curve is the average. The requirement of Table 1 is plotted as the dashed orange line. 


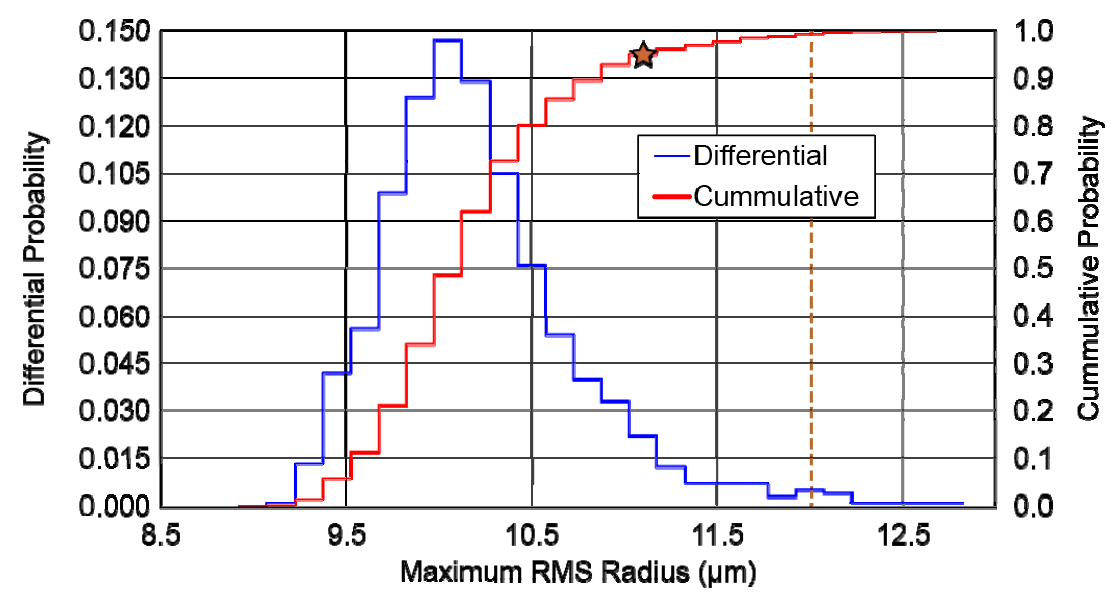

Figure 20: Statistics from the Monte Carlo simulations for the tolerancing of the red channel of the spectrograph. The blue curve is the differential probability distribution and the red is the cumulative distribution.

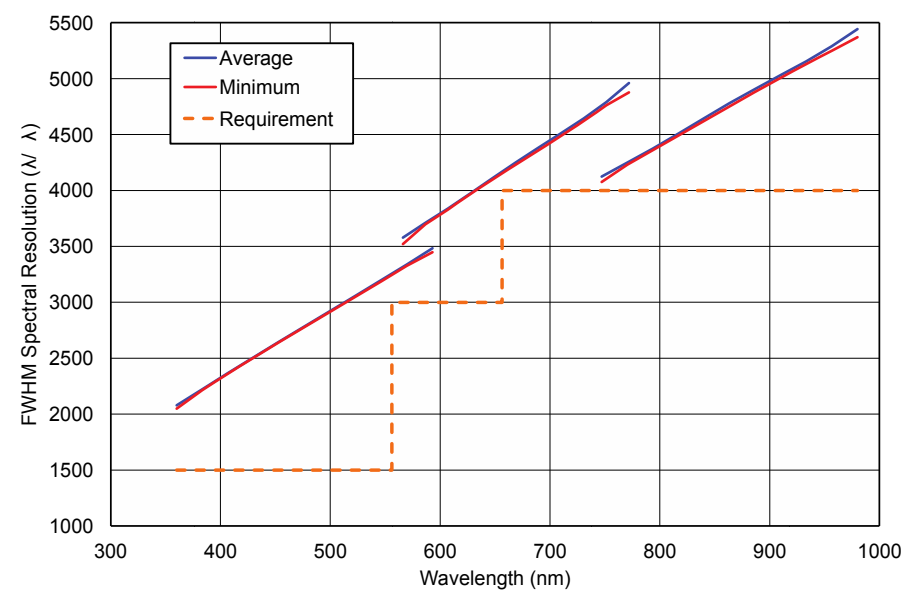

Figure 21: The minimum and average FWHM spectral resolution versus wavelength for the simulated spectrograph. The red curve is the minimum spectral resolution, the blue curve is the average resolution and the dashed orange curve is the requirement.

The $50 \%$ encircled energy diameter for the simulated spectrograph is shown in left of Figure 22 . The red curve is the maximum for all fibers and the blue curve is the average. The simulated spectrograph meets the requirement of $<50 \mu \mathrm{m}$ from Table 1 for all wavelengths and all channels. The $95 \%$ encircled energy diameter for the simulated spectrograph is shown in right of Figure 22. The red curve is the maximum for all fibers and the blue curve is the average. The simulated spectrograph meets the requirement of $<110 \mu \mathrm{m}$ from Table 1 for all wavelengths and all channels.

The throughput of the spectrograph, from the fiber to the CCD, was also simulated. The transmission of the cameras was estimated using the published vendor data and Zemax ${ }^{\circledR}$. The antireflection coatings on the lenses were those measured on the sample coatings by Winlight. The measurements of the collimator mirror reflectance the dichroic performance were used. Grating e ciencies were the measurements by LBNL. The measured quantum efficiency of the CCD detectors was used. The estimated throughput is shown in Figure 23. The blue, red and black curves are for the blue, red and NIR channels respectively. The green curve is the sum of the channel throughputs and the dashed blue curve is the requirement from Table 1. The throughput meets the requirement for all wavelengths.

Two preliminary ghost studies have been completed on the spectrograph. The results show that all ghosts are compliant with the specification of an irradiance of $<10^{-5}$ of the incident irradiance. A complete ghost and stray light 
analysis of the spectrograph was performed with the assistance of Photon Engineering (Tucson AZ). This study was used to define baffles, surfaces and the surface roughness and particulate requirements for the optical surfaces.
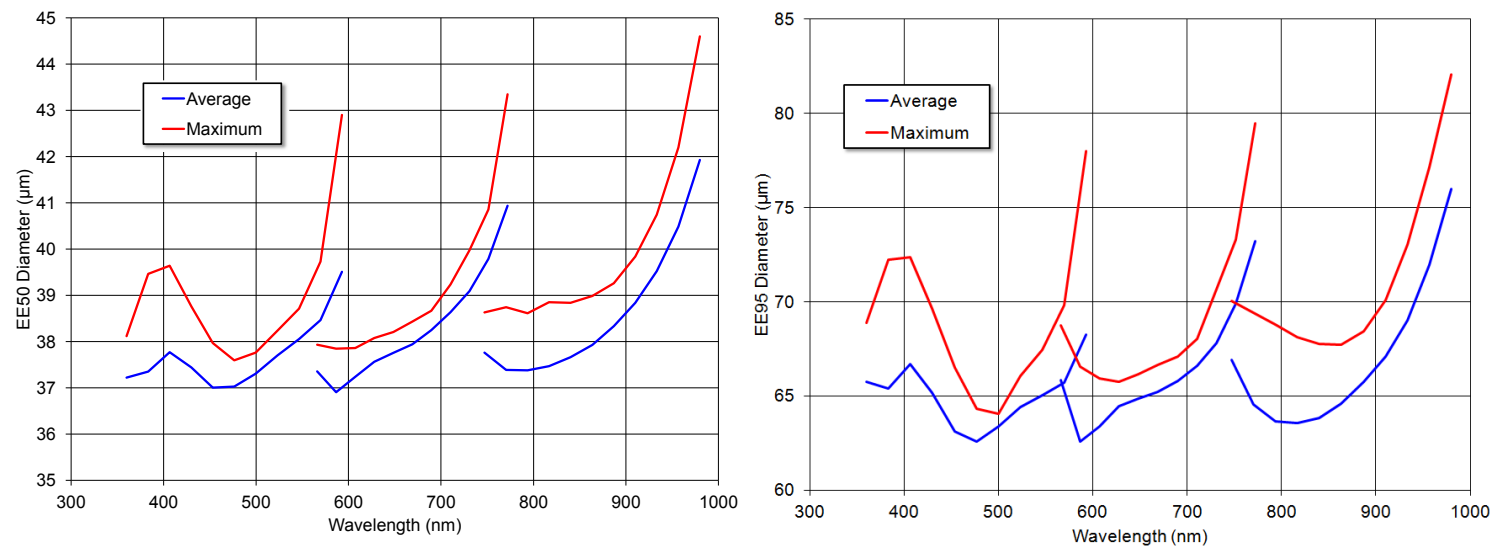

Figure 22: Left: The average (blue) and maximum (red) diameters of the 50\% encircled energy versus wavelength for the simulated spectrograph. The requirement is $<50 \mu \mathrm{m}$. Right: The maximum diameter of the $95 \%$ encircled energy versus wavelength for the simulated spectrograph. The requirement is $<110 \mu \mathrm{m}$.

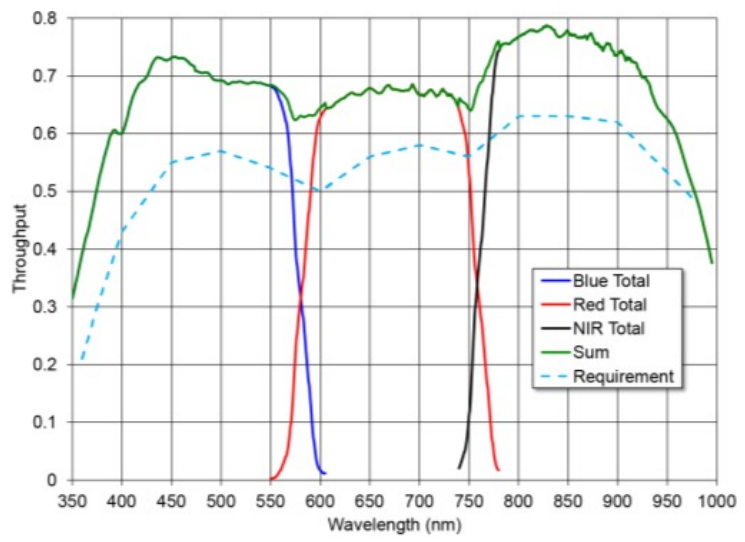

Figure 23: The estimated throughput of the spectrograph. The blue, red and black curves are for the blue, red and NIR channels respectively. The green curve is the sum of the channel throughputs and the dashed blue curve is the requirement.

\subsection{Spectrograph Integration and Testing}

The first spectrograph has been successfully fabricated, integrated and tested by the Aix Marseille Universities at Winlight Systems, as described in these Proceedings ${ }^{7}$. The spectrograph production consists of testing and integration of major assemblies, the camera plus VPH mount, the collimator and Hartmann unit, the dichroic, the cryostat, the shutters, and the baffles and covers.

The cameras are first joined with their VPH gratings at an angle to maximize throughput. The camera assembly is then aligned by measuring the focal surface point spread function (PSF) with reference to the cryostat interface. A special camera test stand uses a collimator in the same configuration as the spectrograph to feed narrow-line spectral fields to the camera unit aperture. The camera output focal plane is then examined with a translating microscope.

The NIR dichroic and collimator are then integrated on a sub-bench that is aligned with a tooling slit that is collocated with the nominal location of the fiber-slit. The camera units are mounted and aligned to the bench, and the camera field is confirmed, as shown in Figure 24.

Cryostats are then attached to the cameras and aligned to each focal plane. The final elements integrated are the shutters, baffles and light covers, as shown in Figure 25. Final acceptance tests include verification of the spectral field, PSF, efficiency, and scattering. 


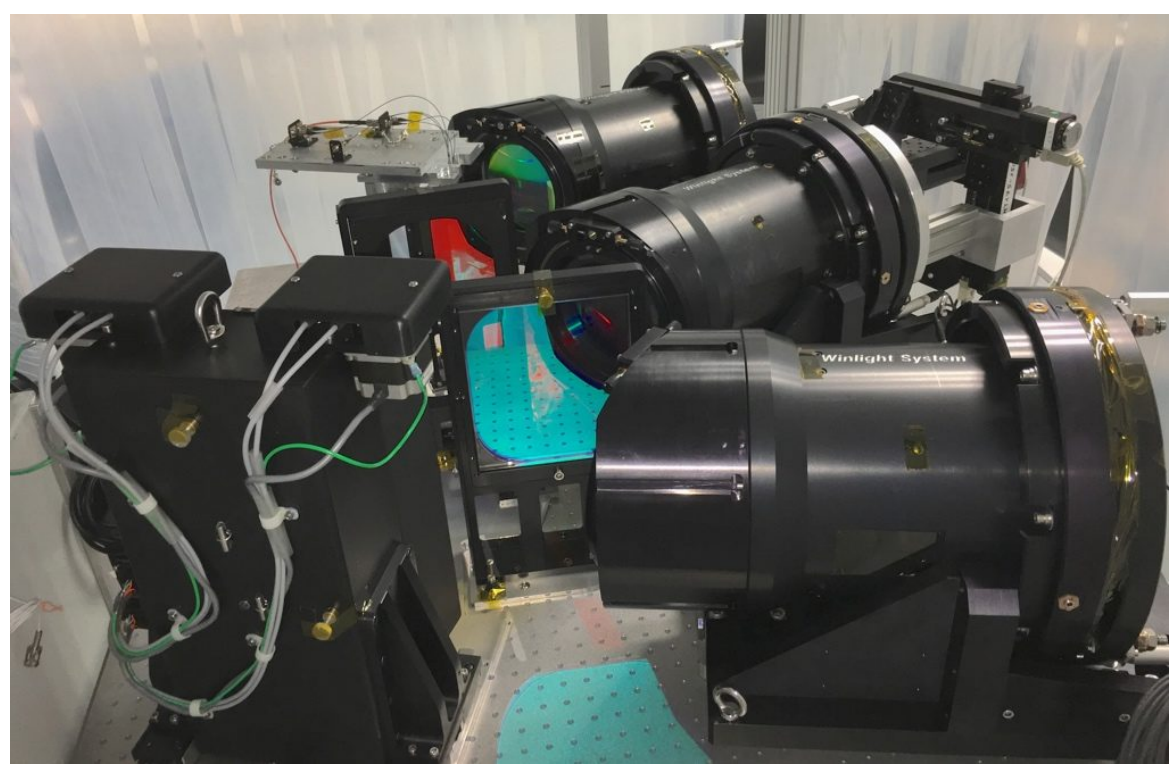

Figure 24: The DESI spectrograph in integrated alignment using a tooling slit source. The cryostats are not attached to the cameras and their focal planes can be measured with a microscope. The dichroic filters are visible (blue and red) and the furthest, NIR channel camera VPH is visible (green). The nearest camera is the red channel. The shutters and baffles are integrated at a later time.

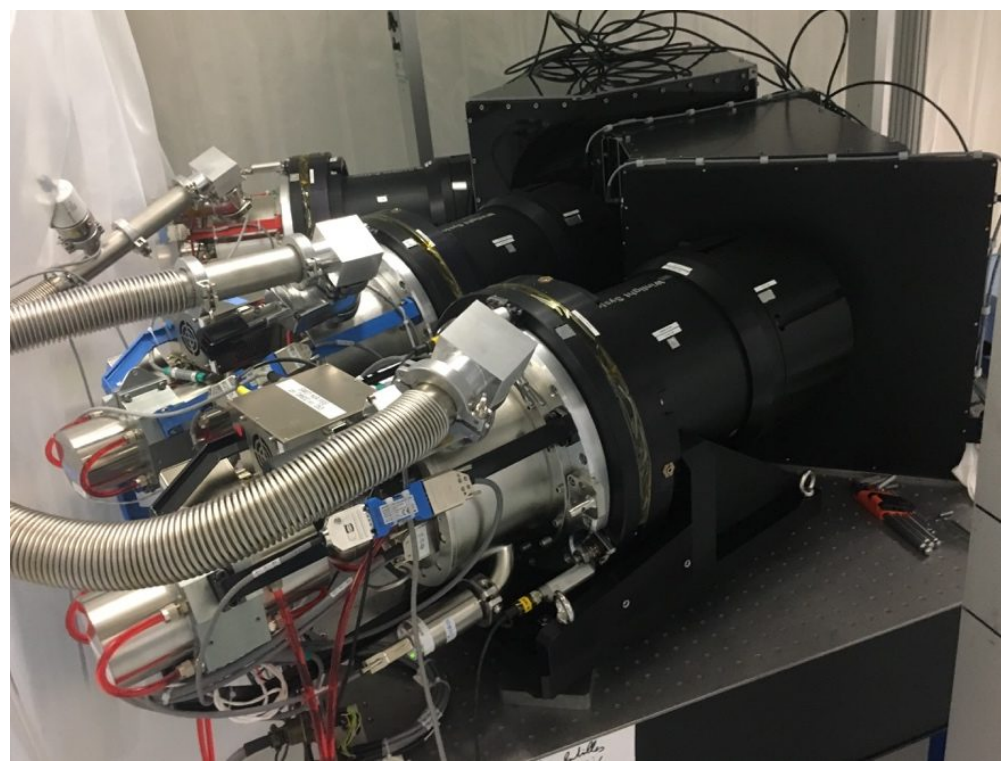

Figure 25: The first completed DESI spectrograph, including the baffles and covers. The cryostat assemblies are foreground.

Following operational, performance, and environmental testing, the spectrographs will then be disassembled in-part and shipped to Kitt Peak. The first spectrograph has arrived at Kitt Peak and the remainder are expected arrive by the early 2019. The spectrographs will be unpacked and reassembled and functionality verified. Test fiber-slit assemblies will be joined to the spectrograph for testing calibration. Then the $\sim 500 \mathrm{~kg}$ spectrographs will be mounted in five stacks of two each in a temperature-controlled enclosure. The science fiber slit and a flat-field illuminator are attached for calibration and test. Sky and dome illumination are used for the science slit sources. The flat-field illuminator produces a nearly uniform continuum source to measure the CCD detectors' spectral flat field. 


\section{CONCLUSIONS}

The first DESI spectrograph meets all of its specifications and has been delivered to Kitt Peak for installation and testing. The production of the remaining nine spectrographs is well underway. They should all be installed at the Mayall telescope by May 2019.

\section{ACKNOWLEDGEMENTS}

This research is supported by the Director, Office of Science, Office of High Energy Physics of the U.S. Department of Energy under Contract No. DE-AC02-05CH1123, and by the National Energy Research Scientific Computing Center, a DOE Office of Science User Facility under the same contract; additional support for DESI is provided by the U.S. National Science Foundation, Division of Astronomical Sciences under Contract No. AST-0950945 to the National Optical Astronomy Observatory; the Science and Technologies Facilities Council of the United Kingdom; the Gordon and Betty Moore Foundation; the Heising-Simons Foundation; the National Council of Science and Technology of Mexico, and by the DESI Member Institutions. The authors are honored to be permitted to conduct astronomical research on Iolkam Du'ag (Kitt Peak), a mountain with particular significance to the Tohono O'odham Nation.

\section{REFERENCES}

[1] Martini, P. et al., " Overview of the Dark Energy Spectroscopic Instrument," Proc. SPIE -110-, xxx (2018).

[2] Tyas, L. et al., "Design and Production of DESI Slit Assemblies," Proc. SPIE -109-, xxx (2018).

[3] Burgh, E.B. et al. "Recombination Ghosts in Littrow Configuration: Implications for Spectrographs Using Volume Phase Holographic Gratings," Proc. Astron. Soc. Pacific 119, 1069-1082 (2007).

[4] Smee, S.A. et al. "The Multi-object, Fiber-fed Spectrographs for the Sloan Digital Sky Survey and the Baryon Oscillation Spectroscopic Survey," Astron. J. 146, 32 (2013).

[5] O'Brien, T. P. et al. "Design of the KOSMOS oil-coupled spectrograph camera lenses," SPIE 9151, 141 (2014).

[6] Honscheid, K. et al, "Status and Early Testing of the DESI Readout and Instrument Control System," Proc. SPIE $110-, \operatorname{xxx}(2018)$.

[7] Perruchot, S. et al. "Integration and testing of the DESI multi-object spectrograph: performance tests and results for the first unit out of ten," Proc. SPIE -105-, xxx (2018). 